\title{
ON EXTENDING CHARACTERS
}

\author{
PAUL MILNES ${ }^{1}$
}

\begin{abstract}
Amplifying modestly on recent work of R. W. Henrichs, we indicate that, while the impossibility of extending a continuous character $\psi$ on a subgroup $H$ of a topological group $G$ to a character on $G$ is often due to algebraic problems, the impossibility of extending $\psi$ to a continuous positive definite function on $G$ can often be viewed as a problem with uniform continuity.
\end{abstract}

Recently R. W. Henrichs [3] proved the following attractive results.

THEOREM 1. Let $G$ be a connected locally compact group which is not the direct product of a vector group and a compact group. Then there is a continuous character on a closed subgroup of $G$ which cannot be extended to a continuous positive definite function on $G$.

(For terminology and further references here, and in the rest of this note, the reader is referred to [2], [3], [5].)

THEOREM 2. Let $G$ be an almost connected locally compact group. Then the following statements are equivalent.

(i) The restriction map $B(G) \rightarrow B(H)$ of Fourier-Stieltjes algebras is surjective for every closed subgroup $H$ of $G$.

(ii) Characters of closed subgroups always have continuous positive definite extensions.

(iii) $G$ is the semidirect product of a vector group $V$ and a compact group $K$ such that $K$ acts on $V$ effectively as a finite group.

These theorems and our treatment of an example on the affine group of the line in [7] prompted us to investigate the possibility that some characters on the subgroups (as in the theorems) might not extend to uniformly continuous functions on the whole group. (We remind the reader that continuous positive definite functions on topological groups are uniformly continuous [5, Theorem 32.4].)

We note first, that it will often be impossible to extend a character of a subgroup to a character of the containing group. For example, such nonextendable characters will always exist on a normal abelian subgroup $H$ of a group $G$ if $H$ is not contained in the center of $G$; thus, if $H$ is a cyclic subgroup of order 3 of the

Received by the editors May 23, 1980.

1980 Mathematics Subject Classification. Primary 43A35.

Key words and phrases. Topological group, positive definite function, character.

'Research supported in part by Natural Sciences and Engineering Research Council of Canada, Grant A7857. 
symmetric group $S_{3}$, the two nontrivial characters on $H$ cannot be extended to characters of $S_{3}$.

On the other hand, if $G$ is a direct product, $G=K \times H$, and $\psi$ is a continuous character on $H$, then defining

$$
\psi^{\prime}(k, h)=\psi(h), \quad(k, h) \in K \times H,
$$

extends $\psi$ to a continuous character on $G$. Also, if $H$ is an open subgroup of $G$ and $\psi$ is a continuous character on $H$, then $p$, defined by

$$
p(g)= \begin{cases}\psi(g), & g \in H, \\ 0, & \text { otherwise, }\end{cases}
$$

is a continuous positive definite function on $G$ extending $\psi[5,(32.43)(a)]$. We note as well the result of Herz [4, p. 94] that, if $H$ is a closed subgroup of a locally compact group $G$ and $p$ is a positive definite function in the Fourier algebra of $H$, then $p$ extends to a positive definite function in the Fourier algebra of $G$.

We come now to our main result and would like to emphasize that, although the hypothesis on the subgroup $H$ is strong, there is no connectivity hypothesis on $G$, which need not even be locally compact.

THEOREM. Let $G$ be a topological group and let $H$ be a normal subgroup of $G$ of the form $H=R^{n} \times T^{m}$, where $R$ and $T$ are the real numbers and the torus, respectively. Then, if the centralizer $Z_{G}(H)$ of $H$ in $G$ is not open, there is a continuous character on $H$ that does not extend to a uniformly continuous function on $G$.

Proof. Since $Z_{G}(H)$ is not open, there is a net $\left\{k_{\alpha}\right\}$ in its complement converging to $e \in G$. Now, for each $\alpha, k_{\alpha} \notin Z_{G}(H)$ means there is a $y_{\alpha}$ in $H$ such that $k_{\alpha} y_{\alpha} k_{\alpha}^{-1} \neq y_{\alpha}$. One sees readily that each map

$$
y \rightarrow k_{\alpha} y k_{\alpha}^{-1}, \quad H \rightarrow H,
$$

must map (the subgroup of $H$ isomorphic to) $R^{n}$ onto $R^{n}$ and $T^{m}$ onto $T^{m}$. It follows then from the form of continuous automorphisms of $T^{m}$ [9] that, taking a subnet of $\left\{k_{\alpha}\right\} \mathrm{f}$ necessary and using the notation

$$
y=\left(y_{1}, y_{2}, \ldots, y_{n}, w_{1}, w_{2}, \ldots, w_{m}\right)
$$

for $y \in H$, we can find a fixed $j \in\{1,2, \ldots, n\}$ such that $\left(k_{\alpha} y_{\alpha} k_{\alpha}^{-1}\right)_{j} \neq\left(y_{\alpha}\right)_{j}$ for all $\alpha$. Then $\psi(y)=e^{i_{j}}$ defines the required character: $\psi$ cannot be extended to a uniformly continuous function on $G$. For, since each map

$$
y \rightarrow k_{\alpha} y k_{\alpha}^{-1}, \quad H \rightarrow H,
$$

is linear on $R^{n}$, we can assume

$$
\left|\left(k_{\alpha} y_{\alpha} k_{\alpha}^{-1}\right)_{j}-\left(y_{\alpha}\right)_{j}\right|=\pi
$$

for all $\alpha$. Then nets $\left\{y_{\alpha}\right\}$ and $\left\{k_{\alpha} y_{\alpha} k_{\alpha}^{-1}\right\}$ are in $H$ and, together with $\left\{k_{\alpha} y_{\alpha}\right\}$, satisfy

$$
\left(k_{\alpha} y_{\alpha}\right) y_{\alpha}^{-1} \rightarrow e, \quad\left(k_{\alpha} y_{\alpha} k_{\alpha}^{-1}\right)^{-1} k_{\alpha} y_{\alpha} \rightarrow e,
$$

while $\left|\psi\left(k_{\alpha} y_{\alpha} k_{\alpha}^{-1}\right)-\psi\left(y_{\alpha}\right)\right|=2$ for all $\alpha$. Thus the values at points in $\left\{k_{\alpha} y_{\alpha}\right\}$ of any uniformly continuous extension of $\psi$ must become arbitrarily close to each of two numbers which are separated by a distance of two. This is impossible and completes the proof. 
Remarks. 1. It is clear the conclusion of the theorem holds in somewhat more general setting. The question arises: if $G$ satisfies the hypothesis of Henrich's Theorem 1 or satisfies the hypothesis, but none of the equivalent conditions of his Theorem 2, does it follow that there is a continuous character on a closed subgroup of $G$ that cannot be extended to a uniformly continuous function on $G$ ?

2. The proof of the theorem shows that, if $\psi$ is the character defined on $\boldsymbol{H}$ and $\boldsymbol{f}$ is any uniformly continuous function on $G$, then $\left\|\psi-\left.f\right|_{H}\right\|_{\infty}>1$. For the affine group of the line and its normal subgroup $R$, the conclusion of the theorem and the one just mentioned appear in [7].

3. It follows from basic uniform space theory that every continuous character (or any right uniformly continuous bounded function) on a subgroup of a topological group $G$ extends to a right uniformly continuous function on $G$ [6]. In the case of the Euclidean group of the plane $G=T \otimes_{\sigma} C$, nontrivial characters of the complex plane $C$ do not extend to uniformly continuous functions on $G$, but do extend to right uniformly continuous distal functions on $G$ [8]. It seems unexpected that right uniformly continuous distal functions can fail to be left uniformly continuous.

We had originally formulated the theorems above for the case where $G$ is a semidirect product, $G=K \otimes_{\sigma} H$, with $K$ acting on $H=R^{n}$. We thank the referee for indicating to us that our proof could be made to work in a more general setting. We also thank him for mentioning the related results in [1] and for devising the following corollary, whose proof uses Theorem 2 quoted at the beginning and a result in [2].

COROLlARY. Let $H=R^{n} \times T^{m}$ be a normal subgroup of a locally compact group $G$. Then the following conditions are equivalent.

(a) The restriction map $B(G) \rightarrow B(H)$ of Fourier-Stieltjes algebras is onto.

(b) Every continuous character of $H$ extends to a uniformly continuous function on G.

(c) $Z_{G}(H)$ is open,

(d) $G \in[S I N]_{H}$.

\section{REFERENCES}

1. M. Cowling and P. Rodway, Restrictions of certain function spaces to closed subgroups of locally compact groups, Pacific J. Math. 80 (1979), 91-104.

2. R. W. Henrichs, Über Fortsetzung positiv definiter Functionen, Math. Ann. 232 (1978), 131-150.

3. $\ldots$ On characters of subgroups, Indag. Math. 41 (1979), 273-281.

4. C. S. Herz, Harmonic synthesis for subgroups, Ann. Inst. Fourier (Grenoble) 23 (1973), 91-123.

5. E. Hewitt and K. A. Ross, Abstract harmonic analysis. I; II, Springer-Verlag, Berlin, 1963; 1970.

6. M. Kătetov, On real-valued functions in topological spaces, Fund. Math. 39 (1951), 85-91; correction 40 (1953), 203-205.

7. P. Milnes, Compactifications of semitopological semigroups, J. Austral. Math. Soc. 15 (1973), 488-503.

8. , Minimal and distal functions on some non-abelian groups, Math. Scand. (to appear).

9. W. Reddy, The existence of expansive homeomorphisms on manifolds, Duke Math. J. 32 (1965), 627-632.

Department of Mathematics, The University of Western Ontario, london, Ontario, Canada N6A 5B9 